\title{
DossIER
}

Problemas ligados ao Álcool

\section{Abordagem terapêutica da dependência alcoólica}

T. SÁ NOGUEIRA, * C. RIBEIRO**

\section{RESUMO}

Objectivos: Apresentar uma abordagem compreensiva e actual do tratamento da dependência alcoólica. É importante que o profissional de Cuidados de Saúde Primários, nomeadamente o médico de família, tenha conhecimento destes novos tipos de tratamento, de modo a estar mais actualizado e, a intervir, sempre que tal seja necessário, junto destes doentes, em articulacão com outros níveis de cuidados.

Metodologia: Revisão numa perspectiva essencialmente clínica, das possiveis abordagens farmacológicas e psicoterapêuticas utilizadas na actualidade.

Resultados: A abordagem terapêutica do paciente alcoólico deverá corresponder a duas fases: a desintoxicação e a desabituação, cujos objectivos são respectivamente, prevenir e tratar a síndrome de abstinência, e ajudar o paciente a organizar a sua vida relacional, social e profissional, prevenindo assim a recaída.

Conclusões: A abordagem mais efectiva da dependência alcoólica, actualmente, consiste na combinação de terapias farmacológicas e psicossociais, tanto na fase de desintoxicação como na de desabituação e reabilitação.

Palavras-chave: Dependência Alcoólica; Abordagem Farmacológica; Intervencões Psicossociais; Álcool; Tratamento.

*Chefe de Serviço de Psiquiatria. Mestre pela Faculdade de Medicina da Universidade de Lisboa.IDT

**Assistente Graduada de Clínica

Geral do Centro de Saúde de Sete

Rios. Docente da Faculdade de Medicina da Universidade de Lisboa

\section{INTRODUCÃ̃o}

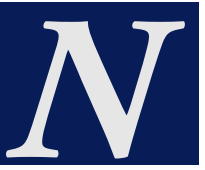

o contexto da classificação dos problemas ligados ao álcool, a OMS considera Consumo de risco, Consumo nocivo e Consumo dependente. $\mathrm{O}$ conceito de consumo de risco (Hazardous) foi criado e introduzido por Babor et al, 1994. ${ }^{1}$

Consumo de risco (Hazardous) ${ }^{2}$ é definido como um padrão de consumo que pode vir a implicar dano físico ou mental, se esse consumo persistir. É um padrão de consumo de muita importância em saúde pública apesar da ausência de alguma perturbação evidente no utilizador.

A intervenção atempada no caso das pessoas cujo consumo é de risco pode prevenir o aparecimento de problemas. De um modo geral, as pessoas não se sentem motivadas, de início, a tomar a decisão de modificar o seu estilo de vida no sentido de reduzir o consumo das bebidas.
Consumo nocivo (Harmful) ${ }^{2}$ define-se como um padrão de consumo que causa danos à saúde, quer física quer mental. Todavia o transtorno não satisfaz os critérios de dependência.

Dependência ${ }^{2}$ define-se como um padrão de consumos constituído por um conjunto de fenómenos fisiológicos, cognitivos e comportamentais, que pode desenvolver-se após repetido uso de álcool. Inclui um desejo intenso de consumir bebidas alcoólicas, descontrolo sobre o seu uso, continuação dos consumos independentemente das consequências, uma alta prioridade dada aos consumos em detrimento de outras actividades e obrigações, aumento da tolerância ao álcool e sintomas de privação quando o consumo é descontinuado.

\section{Diagnóstico da dependência} alcoólica (DSM IV) ${ }^{3}$

- Um forte desejo ou compulsão para consumir álcool;

- Comprometimento da capacidade de controlar o comportamento de uso do álcool em termos do seu início, término ou níveis de uso;

- Um estado fisiológico de abstinência e quando o uso de álcool é reduzido ou interrompido;

- Evidência de tolerância;

- Redução ou abandono de prazeres com o uso de álcool;

- Uso persistente com consequências nocivas

Quando deve o médico de família intervir perante problemas ligados ao álcool em Cuidados de Saúde Primários?

O médico de família deve intervir no: 
- Consumo de risco

- Consumo nocivo

- Dependentes Alcoólicos se

- Sem comorbilidade psiquiátrica

- Sem complicações clinicas

- Sem limitações sociais

\section{Tratamento da desintoxicação} alcoólica ambulatória

- Ausência de complicações graves do ponto de vista orgânico (antecedentes de delirium, psicopatologia comórbida, estado orgânico comprometido, etc.)

- Compromisso do paciente:

- De não beber durante a desintoxicação

- De não sair do domicílio familiar e não realizar actividades de risco

- Presença de um familiar sem problemas de adição que seja responsável por administrar a medicação e supervisionar o tratamento

- Supressão de bebidas alcoólicas no domicílio enquanto durar a desintoxicação

- Vigilância médica com monitorização

\section{Quando deve o médico de familia} referenciar para outro nivel de cuidados?

Dependentes alcoólicos:

- Com tratamentos anteriores

- Risco de privação moderada a severa

- Comorbilidade psiquiátrica

- Sem suporte familiar

- Uso regular de outras substâncias aditivas

Qual o papel dos Cuidados de Saúde Secundários em relação à abordagem da dependência alcoólica?

Critérios para tratamento em regime ambulatório:

- Doente com consciência da doença alcoólica

- Motivado

- Que aceita a abstinência total definitiva como condição da sua recuperação global
- Saúde física, psíquica e social aceitáveis

- Possibilidade de permanecer ligado ao programa terapêutico

Critérios para tratamento em regime de internamento:

- Complicações médicas/psiquiátricas agudas: relacionadas com a intoxicação alcoólica ou com a privação

- Complicações médicas/psiquiátricas crónicas: descompensação de doenças crónicas

- Em relação com o programa de tratamento propriamente dito: para lograr a abstinência com a desintoxicação protegida e como início de desabituação

- Antecedentes recentes de recaídas em meio ambulatório

- Ausência de apoio familiar

\section{Abordagem Terapêutica da Dependência AlCoólica}

\section{Objectivo}

O objectivo da abordagem dos doentes alcoólicos tem por fim a sua recuperação integral, no sentido físico, mental e relacional.

Essa recuperação pressupõe a extinção da conduta dependente e a extinção da conduta dependente implica a desintoxicação e a manutenção de uma abstinência total e definitiva.

Deve contemplar as caracteristicas individuais e de vulnerabilidade de cada doente

A abordagem da dependência consiste em três fases:

\section{Fase de Contacto ou Preparatória}

Para motivar o doente, há que começar por:

- Desculpabilizá-lo

- Fazer-lhe ver a possibilidade de uma mudança

- Mostrar-lhe o ganho que advirá dessa mudança 
- Reforçar-lhe a auto-estima

\section{Fase de Desintoxicação}

Essencialmente farmacológica, para prevenção ou tratamento da síndrome de privação.

\section{Fase de Desabituação ou de EstabiLIZAÇÃo}

- Tratamentos farmacológicos da apetência para o álcool,

- Intervenções psicossociais

\section{Fase de Contacto ou Preparatória}

No que se refere à fase inicial da dependência alcoólica é importante motivar o paciente para que ele aceite as condições do tratamento. Uma das principais dificuldades que aparece nesta fase é a falta de motivação para iniciar o tratamento. Neste sentido é essencial uma adequada avaliação que nos permita saber qual o estado cognitivo de mudança em que o doente se encontra. $\mathrm{O}$ modelo descrito por Prochaska y Di Clemente $^{4}$ pode ser de utilidade, já que em cada estádio se propõem objectivos terapêuticos definidos. ${ }^{1}$

\section{Ciclo da Mudança ${ }^{4}$}

Prochaska e Di Clemente descreveram um conjunto de etapas que decorrem no processo de mudança de comportamentos. Essas etapas são as de pré-contemplação, contemplação, preparação, acção e manutenção. Existe ainda a recaída que corresponde ao retorno a uma etapa anterior à acção.

Neste modelo a mudança é um processo com várias fases e a motivação para mudar vai crescendo gradualmente. O profissional de saúde será mais eficaz se identificar a fase em que se encontra o utente e ajustar a sua intervenção às características dessa fase. ${ }^{5}$ As várias fases também podem ser ilustradas como patamares de uma "escada". As recaídas podem ocorrer em qualquer um dos "degraus", num processo com avanços e recuos até chegar à meta que é a mudança.

É possivel encontrar utentes que não têm consciência do problema e não estão motivados para mudar (pré-contemplação). O objectivo terapêutico será mostrar a possivel relação existente entre o comportamento e suas consequências.

A fase seguinte é marcada pela insegurança e ambivalência face à mudança. O utente não está certo de querer mudar o seu comportamento (contemplação) pelo que se deverá facilitar a análise das vantagens e desvantagens do mesmo.

A terceira fase corresponde à preparação para a mudança. Uma decisão foi tomada, a sua implementação foi planeada e começa a ser executada (preparação). O papel do profissional de saúde será o de destacar acções necessárias para a mudança.

Na quarta fase o utente mudou o comportamento (acção). Será apoiado para que a mudança seja efectiva.

A quinta fase é de manter esse comportamento (manutenção). É necessário enfrentar situações de risco e contar por vezes com o suporte do profissional de saúde no sentido de reforçar a manutenção.

Mas a recaída também pode acontecer. A recaída pode ser mais um passo no sentido da mudança efectiva, beneficiando da experiência já realizada e devendo o técnico de saúde valorizar o que houve de positivo durante o periodo em que houve mudança de comportamento, ou seja, durante o tempo em que o doente conseguiu estar abstinente.

\section{Preparação da ABSTINÊNCIA}

Será importante disponibilizar, seja para o doente seja para a família, a informação necessária, que deverá ser:

- Clara;

- Didáctica;

- Não culpabilizante;

- Que incida sobre o consumo e suas consequências; 
- Que mostre a possível reversibilidade, no sentido que todo o percurso seja conhecido e esclarecido, para que o doente e a família possam comprometer-se com o processo terapêutico.

Reconhecendo-se ele próprio como doente, vencida a negação e portanto, motivado para a abstinência, dá-se-lhe a conhecer como o poderá fazer, quer seja em Cuidados de Saúde Primários ou em Cuidados de Saúde Secundários.

\section{Fase de Desintoxicação}

O processo de desintoxicação do paciente alcoólico implica a imediata e total supressão do álcool, e a prevenção ou tratamento da sindrome de abstinência alcoólica (SAA).

O objectivo primordial da desintoxicação é evitar os sintomas de privação causados pela falta do álcool. Esses sintomas de privação resultam da hiperexcitabilidade do sistema nervoso central (inquietação, agitação e convulsões) da hiperactividade do sistema nervoso autónomo (náuseas, vómitos, taquicárdia, tremores, hipertensão) e de alterações cognitivas. ${ }^{6}$

Na ausência de terapêutica, a evolução dos sintomas de privação pode ocorrer em três etapas:

As primeiras $24 \mathrm{~h}$ subsequentes à última ingestão alcoólica cursam com um quadro de SAA de grau leve (ansiedade, inquietação, náuseas, insónias, sudação, suores facial, HTA, taquicárdia flutuante e tremores).

A segunda etapa de grau moderado desenvolve-se entre as $24 \mathrm{e} 72 \mathrm{~h}$ e apresenta-se com inquietação marcada, agitação, tremores moderados, diaforese, náuseas, vómitos, anorexia, diarreia, taquicárdia, HTA; podem juntar-se alterações da percepção, ou alucinações visuais ou auditivas; desorientação, confusão e convulsões generalizadas.

A terceira etapa pode aparecer entre as $72 \mathrm{~h}$ e $96 \mathrm{~h}$ com febre, HTA, taquicárdia, sudação profusa e tremores generalizados). Quando a gravidade é extre- ma e o doente não recebe o tratamento médico devido, a presença de outras patologias concomitantes (Traumatismo crânio encefálico, doença cardiovascular, infecções, pneumonias por aspiração ou alterações hidro-electroelectroliticas) $^{7}$ pode levar à morte.

O tratamento medicamentoso na desintoxicação consiste em sedação, hidratação e vitaminoterapia.

\section{BENXODIAZEPINAS}

A fisiopatologia e a neurobiologia do Síndrome de Abstinência Alcoólica justifica a utilização de fármacos com actividade Gabaérgica, como são as BZD. ${ }^{\mathbf{8 , 5 0}}$

As BZD e, especialmente as de semi-vida longa são fármacos de primeira eleição para o tratamento da SAA.9,49,50

As BZD de vida longa são usadas para prevenir as convulsões e o delirium da SAA (Diazepam - 30 a 80mg/ /dia, Clorazepato dipotássico - 50 a 200 $\mathrm{mg} /$ dia) enquanto que as de semi-vida curta e média (Oxazepam - 50 a 150 $\mathrm{mg} /$ dia, Lorazepam - 3 a 15mg/dia) serão preferiveis em doentes idosos, em que a sobre-sedação deve evitar-se, e naqueles com importante compromisso hepático. ${ }^{6,49,50}$

As BZD podem dar origem a um sobre-consumo e a uma dependência, mas por este motivo, não contra-indicam em nada a sua prescrição no tratamento da abstinência. Incitam sim a limitar a sua prescrição no tempo e a contra-indicar o seu uso na prevenção das recaídas.

As doses de BZD devem ser individualizadas, em função dos seguintes factores: gravidade da sindrome de abstinência, comorbilidades médicas e história de convulsões e delirium.

\section{TIAPRIDE}

É um antagonista dos receptores dopaminérgicos, de acção rápida, biodisponibilidade absoluta, distribuição corporal rápida, $70 \%$ é encontrado na urina. Tem bom efeito sobre os tremores, a 
agitação e as alucinações; tem efeito ansiolitico discreto; carece de poder anticonvulsivante; é eficaz na desintoxicação e não oferece risco, tendo diversos estudos demonstrado a sua eficácia e segurança no tratamento do SAA; pode utilizar-se por via e.v. ou i.m. na dose de 400 a $1.200 \mathrm{mg} / \mathrm{dia}$, e por via oral $800 \mathrm{mg}$ por dia. Demonstrou ainda a sua eficácia mediante associação de outros fármacos de primeira linha especialmente carbamazepina. ${ }^{\mathbf{1 0 , 1 1 , 4 9}}$

\section{Carbamazepina}

É eficaz e bem tolerada na abstinência - duração 4-5 dias em doses de 400-$800 \mathrm{mg}$-é uma alternativa às BZD, pois apresenta menos potenciação dos efeitos cognitivos do consumo do álcool e não tem os efeitos de dependência ou tolerância das BZD; é anti-convulsivante, podendo ter um efeito anti-Kindling, que impede a progressiva gravidade dos sucessivos episódios de SAA. No entanto, apresenta limitações relacionadas com possiveis e graves complicações hepáticas e hematológicas, que poderão ser ultrapassadas pelos novos anti-convulsivantes. ${ }^{12,13,14,49,51}$

\section{Hidratação e Vitaminoterapia}

Deve sempre iniciar-se um aporte de vitaminas do complexo B, especialmente a tiamina. Se estiver presente um défice de tiamina, é preferivel a administração parentérica porque a via oral tem uma absorção limitada; 2 ampolas de $100 \mathrm{mg} /$ dia nos primeiros dias de desintoxicação, podendo posteriormente continuar per os. A deficiência em tiamina pode levar a situações clínicas complicadas (síndrome de Wernicke-Korsakoff) sobretudo após administração de soros glucosados, sem reposição prévia de tiamina. ${ }^{\mathbf{1 5}, 49}$

As vitaminas B6, B9 e B12 são frequentemente prescritas, assim como o Ácido Fólico e a Vitamina C.

É importante a vigilância do ionograma, para posterior correcção do $\mathrm{Na}, \mathrm{K}$ e Mg. A vigilância cardíaca é essencial devida ao risco de arritmias provocadas por severas perturbações hidro-electrolíticas, as quais são corrigidas por $\mathrm{KCl}$ e SO4Mg.

Atenção às reposições de $\mathrm{Na}$, que não deverão ser bruscas, pois podem originar graves doenças desmielinizantes (Mielinose Central Pôntica). ${ }^{16}$

\section{Fase de Desabituação ou de Estabilização}

Esta fase consiste em manter a situação de abstinência, lograda pela desintoxicação. Será a altura de actuar sobre múltiplos aspectos do doente e do seu ambiente, com a finalidade de consolidar o comportamento de não consumo de álcool. É um processo complexo que requere um tratamento estruturado, levado a cabo coordenadamente entre os CSP e os niveis mais especializados. 0 tratamento apoia-se sobre a terapêutica farmacológica, a psicoterapia, a intervenção social e os grupos de auto-ajuda. Os objectivos do tratamento de desabituação e prevenção de recaídas são a extinção do comportamento condicionado de procura e consumo de álcool, a aquisição de consciência de dependência, a manutenção da motivação ao longo de todo o processo, a reorganização das actividades do doente com procura de alternativas, a prevenção de recaídas e o tratamento da psicopatologia associada.

Sendo o Alcoolismo uma doença da química cerebral (hipóteses glutamatérgica, opióide, serotoninérgica e outras) com predisposição hereditária e desencadeada por factores psicológicos e sociais, desenvolveu-se a introdução de novos agentes terapêuticos que permitem uma abordagem mais compreensiva dos alcoolismos do ponto de vista farmacológico:

\section{Fármacos Anti-Craving}

São fármacos que diminuem o desejo e a compulsão para beber e dessa forma, 
reduzem o número de recaídas, e quando estas aparecem, ajudam a voltar à abstinência mais facilmente. Por tal motivo, devem manter-se durante a recaída, e ser administrados durante um período de 6-12meses.

Acamprosato - É o acetilhomotaurinato de cálcio, relacionado com a taurina, o GABA e o glutamato; actua, repondo a actividade gabaérgica normal, diminuída pelo consumo crónico de álcool, e diminuindo o estado de hiperfunção da neurotransmissão excitatória glutamatérgica, através do qual aliviaria o estado de hiperexcitabilidade residual do SNC. Esta neurorregulação reduziria o craving de álcool. A dose habitual é de 2 comprimidos três vezes ao dia; a duração do tratamento é de 6-12 meses, associada a outros fármacos; está contra-indicado na insuficiência renal; não interage com outros fármacos nem com o álcool. Produz uma melhoria na taxa de abstinência e nos dias acumulados de abstinência de bebidas alcoólicas. ${ }^{17,18,51,52}$

Naltrexona -É um antagonista parcial dos receptores opióides. O álcool provoca aumento da actividade opióide, a qual origina aumento da actividade dopaminérgica no núcleo accubens. Esta é a base da capacidade do álcool para produzir reforço e provocar um aumento do desejo de continuar a consumir. Através do bloqueio dos receptores opióides, os consumos iniciais de álcool perderiam a sua capacidade de reforço, e não se daria a perda de controlo, que levaria a um consumo massivo. Pode iniciar-se o consumo de naltrexona mesmo com o álcool, e juntamente com o acamprosato, tiapride e ISRS. Duração de 6-12meses.Dose-50mg/dia. Contra-indicações: gravidez, amamentação, dependência activa a opiáceos e hepatopatias moderadas a graves.Diminui o número de dias de consumo, diminui a tendência a voltar a beber, reduz a recaída e reduz o craving. ${ }^{19,20,48,52}$.

$\mathrm{O}$ ondansetron e o topiramato tam- bém reduzem o craving. No entanto serão descritos mais adiante, por pertencerem a determinados grupos de medicamentos.

\section{Fármacos Antidopaminérgicos}

Tiapride - É um antagonista dos receptores dopaminérgicos que pertence ao grupo das benzamidas. Além de estar indicado no tratamento do SAA, é também útil na prevenção de recaídas do álcool. ${ }^{21}$

\section{FÁrmacos Serotoninérgicos}

Os doentes alcoólicos apresentam níveis de serotonina (5-HT) anormalmente baixos. Assim, estes fármacos tornam-se úteis, não só para o tratamento do alcoolismo, como para o tratamento dos sintomas depressivos. A redução do consumo é próxima dos $30 \%$ e está relacionada com a diminuição do desejo de consumir.

Fluoxetina e Fluvoxamina - Têm-se mostrado úteis na redução do consumo de álcool entre 30-50\% e aumentam os dias de abstinência nos alcoólicos, mesmo na ausência de depressão. A primeira nas doses de 40-80mg/dia é eficaz para diminuir o desejo e o consumo de álcool. A segunda, nas doses de $100 \mathrm{mg} /$ dia, tem um perfil sedativo e ansiolitico mais marcado. ${ }^{22,51}$

Buspirona - Agonista do receptor 5HT-1A; ansiolítico de tipo não benzodiazepínico, que exerce o seu efeito bloqueando os receptores serotoninérgicos. Tem um baixo potencial de abuso e não cria sedação nem alterações motoras quando interage com o álcool. Pode empregar-se quando as BZD estão contra-indicadas. Doses de 20-30mg/d, repartidos em três tomas. É um fármaco que se pode administrar uma vez finalizado o tratamento de desintoxicação. ${ }^{23,24}$

Ondasentron - antagonista dos receptores 5HT3, reduz o álcool e o craving em doentes alcoólicos de início precoce. ${ }^{13,25,26}$ Doses de 0,5mg/dia; é usado 
a nivel hospitalar no tratamento sintomático dos vómitos secundários ao tratamento com radioterapia e quimioterapia. É mais útil nos alcoólicos jovens. Sertralina - Além de inibir o transportador de serotonina, bloqueia a recaptação de dopamina; em doses de 50-200mg/dia mostrou uma diminuição do consumo. ${ }^{23,24,51}$

Citalopram - É um inibidor muito selectivo de recaptação de serotonina; doses de $20-40 \mathrm{mg} /$ dia. $^{23,24,51}$

\section{FÁRMAcos ANTI-EPILÉPticos}

A carbamazepina e o valproato são eficazes para o tratamento da desintoxicação alcoólica. Os novos anti-convulsivantes (gabapentina, topiramato, lamotrigina, oxcarbazepina) estão também a ser estudados no craving e perturbações psiquiátricas associadas. Podem ser úteis em alcoólicos com perturbações da personalidade, ou em indivíduos impulsivos. ${ }^{13,51}$

Topiramato - Há estudos concludentes quanto à redução do craving nos alcoólicos, manifestando-se numa redução de consumos diários, diminuição do desejo de beber, redução dos sintomas de abstinência tardia e também da taxa de recaídas. Doses de $25 \mathrm{mg}$ duas vezes ao dia, aumentando progressivamente até chegar aos 200-300mg/dia. ${ }^{27,28,51,52}$

\section{Fármaco Interditor ou Aversivo}

Dissulfiramo - É um fármaco cuja missão é dissuadir o consumo de álcool, de uma forma voluntária; cobre os primeiros tempos de abstinência e reforça a decisão da mesma. Não suprime o desejo, pelo que se pode associar ao acamprosato, naltrexona e outros. Bloqueia o metabolismo do álcool que consequentemente dá lugar a uma intoxicação por acetaldeído. Doses de 125-250mg administradas por um co-responsável ${ }^{29,51,52}$ e após preenchimento de um consentimento informado assinado pelo doente. Sintomas que aparecem quando se consome álcool: ${ }^{30,49,51,52}$ vaso- dilatação generalizada, cefaleias, taquicárdia, hipotensão ortostática, sudação, vómitos, dispneia, visão turva, vertigem, obnubilação, convulsões, disfunção hepática, arritmias, enfarte do miocárdio e morte, nos casos mais graves. Tratamento sintomático, com anti-histamínicos, corticosteróides e assistência hospitalar. Devem estar avisados para não consumirem ou manipularem substâncias que contenham álcool (cerveja "sem álcool", vinagre, molhos, perfumes, desodorizantes, after-shaves). ${ }^{30}$

São contra-indicações absolutas para o uso de dissulfiramo: gravidez, psicose, reacção tóxica anterior (psiquiátrica, neurológica, hepática ou cardiovascular). São contra-indicações relativas: polinevrite, nevrite óptica, diabetes, insuficiência hepática, afecções dermatológicas, cardiovasculares e cerebrovasculares, alterações do estado geral, epilepsia e perturbação afectiva major, atraso do desenvolvimento intelectual ou deterioração mental ligada à idade, anestesia geral, nefropatia, tuberculose evolutiva, beta-bloqueantes, vasodilatadores, simpaticomiméticos, IMAO, antidepressivos tricíclicos, antipsicóticos. Efeitos secundários: hepatite, neuropatias, episódios psiquiátricos, problemas dermatológicos e gastrointestinais, diminuição da libido, aumentos da TA e do colesterol. ${ }^{31,5152}$

\section{Indicações preferenciais do}

Dissulfiramo $^{31,51}$

Características favoráveis

- Homens > 40 anos

- Traços compulsivos com capacidade de insight

- Estabilidade familiar e social

- Longa história de abuso de álcool com poucas recaídas

- Ausência de depressão ou psicose

- Resposta positiva a tratamentos anteriores

- Motivação

- Frequência de Delirium Tremens

- Contactos com associações de auto- 


\section{DOSSIER}

Problemas ligados ao Álcool

-ajuda

- Transfer positivo em relação ao terapeuta

- Associação a tratamento comportamental e cognitivo

- Abstinência recente com stresse severo

\section{Características desfavoráveis}

- Mulheres jovens

- Traços sociopáticos

- Isolamento social

- Curta história de abuso de álcool com recaídas frequentes

- Presença de depressão ou psicose

- Resposta negativa a tratamentos anteriores

- Ambivalência quanto ao tratamento e abstinência

- Menos antecedentes de Delirium Tre mens

- Menos contactos com associações de ajuda

- Ausência de relação sólida e estável com o terapeuta

\section{INTERVEnC̄ões PsicossociaIS}

O tratamento da dependência alcoólica é feita no contexto de uma abordagem multidisciplinar com uma dimensão biológica, psicológica e social cujo objectivo é o de manter a abstinência e prevenir as recaídas, melhorar a qualidade de vida e reduzir a possivel comorbilidade psiquiátrica do doente alcoólico.

A Associação Americana de Psiquiatria ${ }^{32}$ recomenda:

- Com segurança clínica confirmada pela evidência: Terapias cognitivo-comportamentais, Abordagem motivacional, Terapia familiar ou de casal.

- Com segurança clínica de menor confirmação pela evidência: Terapias de Grupo,Técnicas facilitadoras dos 12 Passos, Terapia psicodinâmica e interpessoal.

Partindo destes dados sustentados pela evidência, faremos uma revisão sumária das abordagens que demonstraram maior eficácia, salientando, no entanto, que a melhor abordagem é aquela que combina o tratamento farmacológico com a abordagem psicológica, tendo em conta sempre a individualidade de cada doente e no contexto de um programa integrado de tratamento.

\section{Terapias Cognitivo-Comportamentais} A maioria dos ensaios clínicos mostra a eficácia deste tipo de intervenção, ${ }^{33}$ ou em combinação com farmacoterapia ${ }^{34}$ no tratamento da dependência alcoólica.

O objectivo deste tipo de terapia é ensinar o doente a identificar situações de risco em relação ao consumo de álcool e ajudar a aumentar o auto-controlo e as aptidões para lidar com estas situações.

Estas técnicas baseadas na teoria da aprendizagem social têm como objectivo aumentar as competências do doente para enfrentar situações de alto risco. As mais utilizadas na prática clínica são: Prevenção de recaídas, treino em competências sociais ou de comunicação, treino na abordagem do craving.

Partem do princípio de que existem vários factores que aumentam a vulnerabilidade do doente alcoólico à recaída: a limitação de recursos no contacto com o stresse, as emoções e situações de risco (em que o álcool e a pressão para o consumo estão presentes) expectativas relacionadas com o álcool e os efeitos positivos, e uma limitada ou reduzida percepção de auto-eficácia.

A maioria destas abordagens faz uma análise dos factores de vulnerabilidade individual na qual deverão estar presentes factores biológicos e psiquiátricos, assim como uma análise de factores de risco relacionados com o consumo de álcool. Pretende-se com este tipo de intervenções que se consigam enfrentar situações de risco mediante a combinação de técnicas de prevenção de recaídas e dos estados de craving. Também 
deverá ser considerado em continuidade o trabalho dirigido para o desenvolvimento de competências sociais que ajude a melhorar as relações familiares e profissionais do indivíduo, aumentando deste modo a percepção de auto-eficácia. ${ }^{35}$ Estes modelos de abordagem resultam preferencialmente em individuos em que não exista grande deterioração cognitiva, pessoal e social. Nos casos com maiores compromissos resultam melhor as abordagens de maior suporte social (terapias de grupo, alcoólicos anónimos).

\section{Prevenção de recaídas}

Uma das técnicas mais utilizadas é a da prevenção da recaída. Nesta modalidade psicoterapêutica, o tratamento é individualizado, abordando variáveis cognitivas, ou seja, os pensamentos do sujeito que determinam o seu comportamento. O sujeito é considerado como o principal agente de mudança, tanto a nível da abstinência como da vida pessoal. ${ }^{36}$

Assim, deve ser apoiado na motivação, na evicção de situações ou lugares onde haja consumo, na utilização de suporte de familiares e/ou amigos, na valorização da mudança de comportamento e no desenvolvimento de comportamentos alternativos.

Neste sentido, podem ser de grande utilidade os programas de treino (assertividade, competências na comunicação, resistência à pressão do grupo...).

No caso de recaída existem programas específicos como o de Marlatt y Gordon. ${ }^{36}$

\section{Treino de competências sociais ou de comunicação}

O objectivo desta técnica é o de adquirir um estilo de comunicação assertiva e eficaz que permita construir e manter relações sociais sem o álcool; de facto trata-se também de um modelo para enfrentar situações de risco. A maior parte dos estudos identificam também resultados positivos com esta técnica ${ }^{37}$ (Botlender 2006) tendo os doentes mantido a abstinência mais tempo, ou seja tendo o primeiro episódio de recaída, pós-tratamento, mais tarde.

\section{INTERVENCÕES MOTIVACIONAIS}

Englobam um conjunto de estratégias orientadas para aumentar a motivação intrínseca do indivíduo num tempo relativamente breve de intervenção.

É uma técnica que se deve utilizar desde o início da intervenção, nomeadamente na fase exploratória com continuidade para a fase resolutiva.

Os elementos essenciais são: a escuta reflexiva e empática, a disponibilização da informação personalizada, a promoção de um discurso auto-motivador e a implicação do doente na responsabilidade de um compromisso terapêutico, aumentando a percepção de auto-eficácia. Este tipo de abordagem é adequada, seja na dependência sem grande compromisso cognitivo, seja no consumo de risco e nocivo. ${ }^{37,38}$ Este tipo de abordagem parece mostrar resultados superiores ao conselho médico clássico e mesmo à terapia cognitivo-comportamental, demonstrando resultados positivos e de baixo custo. ${ }^{39}$

\section{Abordagem de Reforco Comunitário}

Outra forma de terapia dentro da linha comportamental, que também tem tido boa resposta em termos de eficácia, é a abordagem de reforço comunitário cujo objectivo é o de conseguir a abstinência mediante a diminuição do reforço positivo associado ao consumo e o aumento do reforço positivo em estado de sobriedade. As componentes deste tipo de intervenção ajustam-se às necessidades individuais, tendo como principal objectivo a construção da motivação para a abstinência. ${ }^{40}$ 


\section{DOSSIER}

Problemas ligados ao Álcool

\section{TERAPIA DE CASAL/FAMILIAR}

Existe uma forte recomendação deste tipo de abordagem no tratamento da dependência alcoólica. ${ }^{41}$

É fundamental o papel da família (favorece ou sofre as consequências da doença e favorece ou dificulta a recuperação do doente) sem esquecer o desenvolvimento de uma possivel patologia do casal ou da família nuclear do paciente com dependência alcoólica.

Pode ser utilizada como método para incluir os familiares do paciente no processo terapêutico como co-agentes de mudança ou para reorganizar sistemas familiares inadequados que possam ter promovido o consumo.

Resumindo, os resultados encontrados neste tipo de terapia têm resultados positivos, nomeadamente no sentido de aumentar o grau de funcionalidade conjugal ou familiar, ou seja, nos casos em que a relação familiar possa estar deteriorada e existam dificuldades na comunicação.

\section{OUtros Tipos de AbORdAGEM}

\section{Terapias Grupais}

O objectivo é de promover as relações interpessoais e de apoio mútuo, aumentando a auto-estima e a motivação.

Os aspectos que se abordam no grupo, e que são considerados como terapêuticos são os seguintes: informação sobre aspectos da doença, desenvolvimento de crença na possibilidade de mudança, aprendizagem de novas competências sociais.

A coexistência de doentes em diferentes fases do tratamento oferece um efeito de modelagem, aumentando o potencial de aprendizagem de estratégias de consciencialização.

Têm um papel importante na prevenção e detecção de recaídas, permitindo a troca de experiências.
Os estudos comparativos entre abordagens individuais e grupais demonstram que existem resultados similares no que diz respeito à abstinência, ainda que, com uma relação custo eficácia superior. ${ }^{42,43}$

\section{Terapia dos Doze Passos}

Existe eficácia neste modelo, particularmente para aqueles doentes que apresentam síndrome de dependência alcoólica severa, um foco afectivo ou cognitivo, preocupação sobre o sentido da vida. O projecto $\mathrm{MATCH}^{44}$ concluiu sobre a efectividade deste modelo nos indivíduos com uma elevada espiritualidade. Contudo, a revisão sistemática Cochrane ${ }^{45}$ conclui que não há estudos experimentais de qualidade metodológica que demonstrem de forma inequívoca a eficácia deste modelo de terapia. Este tipo de abordagem faz-se em contexto grupal e enfatiza a abstinência absoluta e o reconhecimento da doença. Prevê ainda uma estrutura de suporte social e modelos de referência, no sentido de prevenir as recaídas. É o modelo usado nos AA (Alcoólicos anónimos) - dá-se apoio ao doente durante os cinco primeiros passos e trabalha-se o compromisso com os AA nos passos seguintes.

Como não existem muitos estudos que comprovem a sua qualidade, não há, por este facto, suficiente evidência científica.

\section{Grupos de Auto-Ajuda}

Os grupos de auto ajuda (Alcoólicos Anónimos, Al-Anon, Al-Ateen, Alcoólicos Reabilitados...) exercem a sua função reforçando a decisão de não entrar em contacto com o álcool. O objectivo principal é a abstinência do álcool, assim como ajudar outros alcoólicos a conseguir a sobriedade. ${ }^{46} \mathrm{O}$ trabalho consiste na realização de reuniões em que um membro fala de algum tema particular ou da sua experiência pessoal com o álcool no sentido de poder 
partilhar com o grupo, iniciando um intercâmbio de experiências construtivo. ${ }^{47}$

As finalidades básicas dos grupos de auto-ajuda seriam as seguintes: motivação para o tratamento e recuperação do doente alcoólico; colaboração na prevenção, reabilitação e reinserção deste tipo de doentes; colaboração com os profissionais das equipas assistenciais a doentes com dependência alcoólica, entre outros.

Alguns aspectos a ter em conta dizem respeito à escassez de estudos controlados sobre a sua eficácia.

\section{CONCLUSÕES}

\section{O Médico de Familia perante a Dependência Alcoólica}

A combinação de terapias farmacológicas e psicossociais corresponde ao tipo de abordagem da dependência alcoólica mais eficaz no momento actual, tanto na fase de desintoxicação como na de desabituação e reabilitação do doente alcoólico.

A principal estratégia para conseguir bons resultados no tratamento será aquela que permita uma maior aderência e cumprimento da terapêutica por parte do doente, com a melhor integração social possível.

A escolha de um fármaco ou de outro, ou a indicação de um ou outro recurso psicoterapêutico, deve fazer-se em função das características do doente, da sua dependência e do seu contexto. É demonstrado pela evidência que os dependentes alcoólicos que receberam tratamento têm melhores níveis de abstinência, melhoram as suas relações familiares e satisfação conjugal, com menos deterioração física, psicológica e social. ${ }^{5}$

A importância deste texto, a nível da medicina geral e familiar, permite à luz da evidência traduzir as linhas mais actuais de intervenção em matéria de dependência alcoólica. Pretende ainda mostrar que quanto mais precoce o tipo de abordagem, menor serão seguramente os níveis de deterioração física, psicológica e emocional do doente alcoólico e, se possível, intervir antes que a dependência se instale. Essa sim, é a função primordial dos cuidados de saúde primários, ou seja, intervir no consumo de risco e nocivo, quando ainda não existam critérios de dependência alcoólica, tendo em vista que se dispõe actualmente de um conjunto de estratégias e técnicas de abordagem eficazes, de acordo com a evidência, e que já foram salientados em outros momentos deste trabalho.

O médico de família deverá saber fazer o diagnóstico diferencial do consumo de risco, nocivo e dependência e, quando estiver perante uma dependência, conhecer bem os critérios que justificam a sua referenciação ou, em situações de dependência mais leve, intervir em ambulatório. Se já houver uma dependência e um acompanhamento em cuidados de saúde secundários é importante que o médico de família (independentemente do tipo de abordagem terapêutica que o doente esteja a fazer) seja o suporte tanto do doente como da sua família no processo de recuperação, estando em articulação sempre que tal seja necessário com as estruturas dos cuidados de saúde secundários, que por sua vez deverão manter o médico de família informado do tipo de abordagem instituída, havendo portanto um encontro de cuidados que além de complementares são seguramente necessários para o bem-estar do doente e suas famílias.

\section{REFERÊNCIAS BIBLIOGRÁFICAS}

1. Babor T, Campbell R, Room R, Saunders $\mathrm{J}$, editors. Lexicon of Alcohol and Drug Terms. Geneva: World Health Organization; 1994.

2. Babor T, Higgins-Biddle J, Saunders J, Monteiro MG. The Alcohol Use Disorders Identification Test: guidelines for use in primary health 


\section{DOSSIER}

Problemas ligados ao Álcool care. $2^{\text {nd }}$ ed. Geneva: WHO Department of Mental Health and Substance Dependence; 2001.

3. American Psychiatric Association. DSMIV-TR Manual de diagnóstico e estatística das perturbações mentais. $4^{\mathrm{a}}$ ed. Lisboa: Climepsi Editores; 1996.

4. Prochaska JO, Di Clemente CC.Towards a comprehensive model of change. In: Miller WR, Heather N, editors. Treating addictive behaviors: processes of change. New York: Plenum; 1986. p. 3-27.

5. Gomes CM. O papel do médico de família na detecção e intervenção nos problemas ligados ao álcool a nível dos Cuidados de Saúde Primários. Rev Port Clín Geral 2004 Jan-Fev; 20 (1): 101-18.

6. Blondell RD. Ambulatory detoxification of patients with alcohol dependence. Am Fam Physician 2005 Feb 1; 71 (3): 495-502.

7. Mayo-Smith MF. Pharmacological management of alcohol withdrawal: a meta-analysis and evidence-based practice guideline. American Society of Addiction Medicine Working Group on Pharmacological Management of Alcohol Withdrawal. JAMA. 1997 Jul 9; 278 (2): 144-51.

8. Holbrook AM, Crowther R, Lotter A, Cheng C, King D. Meta-analysis of benzodiazepine use in the treatment of acute alcohol withdrawal. CMAJ 1999 Mar 9; 160 (5): 649-55.

9. Hodges B, Mazur JE. Intravenous ethanol for the treatment of alcohol withdrawal syndrome in critically ill patients. Pharmacotherapy 2004 Nov; 24 (11): 1578-85.

10. Soyka M, Schmidt F, Schmidt P. Efficacy and safety of outpatient alcohol detoxification with a combination of tiapride/carbamazepine: additional evidence. Pharmacopsychiatry 2006 Jan; 39 (1): 30-4.

11. Gartenmaier A, Pelzer E, Soyka M. Treatment of alchol withdrawal syndrome with combined carbamazepine and tiapride in a patient with probable sleep apnoe syndrome. Pharmacopsychiatry 2005 Mar; 38 (2): 96-8.

12. Malcolm R, Myrick H, Brady KT, Ballenger JC. Update on anticonvulsivants for the treatment of alcohol withdrawal. Am J Addict 2001; 10 Suppl: 16-23.

13. Ait-Daoud N, Malcolm RJ Jr, Johnson BA. An overview of medications for the treatment of alcohol withdrawal and alcohol-dependence with an emphasis on the use of older and newer anticonvulsivants. Addict Behav 2006 Sep; 31 (9): 1628-49.

14. Zullino DF, Khazaal Y, Hättenschwiler J, Borgeat F, Besson J. Anticonvulsivants drugs in the treatment of substance withdrawal Drugs Today (Barc.) 2004 Jul; 40 (7): 603-19.
15. Thomson AD, Marshall EJ. The treatment of patients at risk of developing Wernicke's encephalopathy in the community. Alcohol Alcohol 2006 Mar-Apr; 41 (2): 159-67.

16. Estruch R, Sánchez-Turet M. Alteraciones del sistema nervioso asociadas al alcoholismo crónico. In: Sánchez-Turet M, editor. Enfermedades y problemas relacionados con el alcohol. Barcelona: Espaxs; 1999. p. 135-49.

17. Mann K. Pharmacotherapy of alcohol dependence: a review of the clinical data. CNS Drugs 2004; 18 (8): 485-504.

18. Bouza C, Magro A, Muñoz A, Amate JM. Efficacy and safety of naltrexone and acamprosate in the tratment of alcohol dependence: a systematic review. Addiction 2004 Jul; 99 (7): 811-28.

19. Swift RM. Effect of naltrexone on human alcohol consumption. J Clin Psychiatry 1995; 56 Suppl 7: 24-9.

20. Anton RF, O'Malley SS, Ciraulo DA, Cisler RA, Couper D, Donovan DM, et al. Combined pharmacotherapies and behavioral interventions for alcohol dependence: the COMBINE study: a randomized controlled trial. JAMA 2006 May 3; 295 (17): 2003-17.

21. Shaw GK, Waller S, Majumdar SK, Alberts JL, Latham CJ, Dunn G. Tiapride in the prevention of relapse in recently detoxified alcoholics. Br J Psychiatry 1994 Oct; 165 (4): 515-23.

22. Cornelius JR, Salloum IM, Ehler JG, Jarrett PJ, Cornelius MD, Perel JM, et al. Fluoxetine in depressed alcoholics: a double-blind, placebo-controlled trial. Am J Psychiatry 1997 Aug; 54 (8): 700-5.

23. Sánchez-Turet M. Tratamento farmacológico del alcoholismo. In: Sánchez-Turet M, editor. Enfermedades y problemas relacionados con el alcohol. Barcelona: Espaxs; 1999. p. 151-166.

24. Paille F. Les sérotoninergiques: indications sélectives en alcoologie. Alcoologie 1997 Sep; 19 (3 Suppl): 353-360.

25. Johnson BA, Roache JD, Ait-Daoud N, Zanca NA, Velásquez M. Ondansetron reduces the craving of biologically predisposed alcoholics. Psychopharmacology (Berl) 2002 Apr; 160 (4): 408-13.

26. Kranzler HR, Pierucci-Lagha A, Feinn R, Hernández-Ávila C. Effects of ondansetron in early-versus late-onset alcoholics: a prospective, open-label study. Alcohol Clin Exp Res 2003 Jul; 27 (7): 1150-4.

27. Rubio G, Ponce G, Jiménez-Arriero MA Palomo T, Manzanares J, Ferre F. Efects of Topiramate in the treatment of alcohol dependence. Pharmacopsychiatry 2004 Jan; 37 (1): 37-40. 28. Johnson BA, Ait-Daoud N, Bowden CL, 
DiClemente CC, Roach JD, Lawson K, et al. Oral topiramate for treatment of alcohol dependence: a randomised controlled trial. Lancet 2003 May 17; 361 (9370): 1677-85.

29. Williams SH. Medications for treating alcohol dependence. Am Fam Physician 2005 Nov 1; 72 (9): 1775-80.

30. Guardia J. Avances en el tratamiento farmacológico del alcoholismo. En: Aizpiri J, Marcos JF, editors. Actualizacion del tratamiento del alcoholismo. Barcelona: Masson; 1996. p. 65-96.

31. Bénard JY, Husson M, Gabriel I, Yarko N, Chaylard PH, Ferrant JP. Indications du Dissulfiram en alcohologie. Alcohologie 1997 Sep; 19 (3 Suppl): 347-52.

32. APA Work Group on Substance Use Disorders. Practice Guideline for the treatment of patients with Substance Use Disorders. $2^{\text {nd }}$ ed. Arlington, VA: APA ; 2005. p. 89-100.

33. Burtscheidt W, Wölmer W, Schwarz R, Strauss W, Gaebel W. Out-patient behaviour therapy in alcoholism: treatment outcome after 2 years. Acta Psychiatr Scand 2002 Sep; 106 (3): 227-32.

34. Monti PM, Rohsenow DJ, Swift RM, Gulliver SB, Colby SM, Mueller TI, et al. Naltrexone and cue exposure with coping and communication skills training for alcoholics: treatment process and 1-year outcomes. Alcohol Clin Exp Res 2001 Nov; 25 (11): 1634-47.

35. Monti PM, Rohsenow DJ. Coping skills training and cue exposure therapy in the treatment of alcoholism. Alcohol Res Health 1999; 23 (2):107-15.

36. Marlatt G, Gordon JR, editors. Relapse prevention: maintenance strategies in the treatment of addictive behaviors. New York: The Guilford Press; 1985.

37. Bottlender M, Köhler J, Soyka M. Effektivitat psychosozialer Behandlungsmethoden zur medizinischen Rehabilitation alcoholabhangiger Patienten. Fortschr Neurol Psychiatr 2006 Jan, 74 (1): 19-31.

38. Miller WR, Rollnick S. Motivational interviewing preparing people for change. $2^{\text {nd }} \mathrm{ed}$. New York:The Guilford Press; 2002.

39. Rubak S, Sandbaek A, Lauritzen T, Christensen B. Motivational interviewing: a systematic review and meta-analysis. Br J Gen Pract 2005; 55 (513): 305-12.

40. Roozen HG, Boulogne JJ, van Tulder MW, van den Brink W, De Jong CA, Kerkhof AJ. A systematic review of the effectiveness of the community reinforcement approach in alcohol, cocaine and opioid addiction.Drug Alcohol Depend 2004 Apr 9; 74 (1): 1-13.
41. McCrady BS, Epstein EE, Kahler CW. Alcoholics anonymous and relapse prevention as maintenance strategies after conjoint behavioural alcohol treatment for men: 18-month outcomes. JConsult Clin Psychol 2004; 72 (5): 870-8.

42. John U, Veltrup C, Driessen M, Wetterling T, Dilling H. Motivational intervention: an individual counselling vs a group treatment approach for alcohol-dependent in-ptients. Alcohol Alcohol 2003 May-Jun; 38 (3): 263-9.

43. Marqués AC, Formigoni ML. Comparison of individual and group cognitive-behavioral therapy for alcohol and/or drug-dependent patients. Addiction 2001; 96: 835-46.

44. Project MATCH Research Group. Matching alcoholism treatments to client heterogeneity: Project MATCH three-year drinking outcomes. Alcohol Clin Exp Res 1998 Sep; 22 (6): 1300-11.

45. Ferri M, Amato L, Davoli M. Alcoholics Anonymous and other 12 sytep programmes for alcohol dependence.Cochrane Database SystRev 2006 Jul 19; 3: CD005032.

46. Chamorro L. Tratamiento del alcoholismo. In: Valbuena A, Alamo C, editors. Avances en toxicomanías y alcoholismo: aspectos conceptuales, farmacológicos, clinico-terapéuticos y médico-legales. Madrid: Universidad de Alcalá; 1997.

47. Edwards G. Tratamiento de alcohólicos. Guía para el ayudante profesional. 2a ed. México: Trillas; 1992.

48. Garcia JB, Brugué MC, Fraile MG. Manual de evaluación y tratamiento de drogodependencias. Barcelona: Ars Medica; 2003.

49. García-Rodríguez JA, Sánchez CL. Manual de estudios sobre alcohol. Madrid: EDAF; 2001.

50. Mack AH, Franklin Jr. JE, Frances RJ. Tratamento del alcoholismo y las adicciones. Barcelona: Masson; 2003.

51. Cobos Peris JCP, Zurián JC, Martínez GC, Valladolid GR. Tratado SET de Trastornos Adictivos. Madrid: Panamericana; 2006.

52. Cordeiro DC, Figlie NB, Laranjeira R. Boas práticas no tratamento do uso e dependência de substâncias. Rio de Janeiro: Roca; 2007.

\section{Endereço para correspondência}

T. Sá Nogueira

Al. Linha Torres, $256-11^{\circ}$ Dto

1750-152 Lisboa 


\section{DOSSIER}

Problemas ligados ao Álcool

THERAPEUTICAL APPROACH IN ALCOHOLIC PATIENTS

Objectives: to present a comprehensive and current approach for the treatment of alcohol dependence. Toprovide the best support to both patients and their families, family physicians should know these kind of interventions and work with the other levels of alcohol dependent care.

Methods: to make a review of the treatment approach of alcohol dependence.

Results: the therapeutic approach to the alcoholic patients involves two stages: detoxification and maintenance of abstinence. In the former, the goals are the prevention and treatment of the withdrawal syndrome. In the latter it is necessary to help the patient to organise his life without alcohol consumption and to prevent relapse.

Conclusions: the most effective approach in the current treatment of alcoholism is the combination of pharmacological and psychosocial therapies, both in the detoxification and in the maintenance and rehabilitation states.

Key words: Alcohol Dependence; Therapeutic Approach; Psicosocial Intervention; Alcohol; Treatment. 